\title{
Reviewers 2018
}

\section{WAFAA ABD EL-RAHIM}

National Research Center, Cairo, Egypt

NURUL ABSER

University of Science \& Technology, Chittagong, Bangladesh

ALI ADIGÜZEL

Mersin University, Mersin, Turkey

DIANA AFIFAH

Bogor Agricultural University, Indonesia

\section{NNABUgWU AgOMUo}

Imo State University Owerri, Nigeria

\section{Cristobal N. AgUilar}

Universidad Autonoma de Coahuila, Coahuila, Mexico

\section{FARAH AHMAD}

Institute of Biomedical Sciences and Research, Dehradun, India

\section{TANSENA AKHTER}

Tairunnessa Memorial Medical College, Gazipur, Bangladesh

\section{MEHRAN ALAVI}

Razi University, Kermanshah, Iran

Ammar Altemimi

So Illinois University, Carbondale, USA

\section{QI AN}

Beijing Forestry University, Beijing, China

\section{AhMAD ARZANi}

Isfahan University of Technology, Iran

\section{KIRIL BAHCEVANDZIEV}

Polytechnic Institute of Coimbra,

\section{RAMON BATISTA}

Universidad Autonóma del Estado de Morelos, Morelos, Mexico

\section{WANDA BIAEA}

Institute of Bioorganic Chemistry, PAS, Poznań, Poland

MiHAELA BRATU

Ovidius University Constanta, Constanta, Romania

\section{GuOHONG CAI}

Purdue University, West Lafayette, USA

\section{Teresa Cegielska-Taras}

Plant Breeding and Acclimatization Institute - National Research Institute, Poznań, Poland

\section{DigantKumar Chapla}

Sardar Patel University, Vallabh Vidyanagar, India

\section{MOHIT CHAWLA}

King Abdullah University of Science and Technology, Thuwal, Saudi Arabia

\section{JEMMY ChRISTY}

Sathyabama University, Chennai, India

\section{Chris DAYTON}

Fats and Oils Processing for Bunge Global Innovation, Urbana, USA

\section{Pradeep Deo}

Queensland University of Technology, Brisbane, Australia

\section{ALBERT J. DIJKSTRA}

Carbougnères, St. Eutrope-de-Born, France

\section{ALEJANDRo S. Escandón}

Instituto de Genética "Ewald A. Favret”, Buenos Aires, Argentina

\section{SAMEH FAHIM}

Minuofia University, Egypt

\section{MOSTAFA FARAJPOUR}

University of Tehran, Iran

\section{ROBERTO FERNANDEZ-LAFUENTE}

Instituto de Catálisis-CSIC, Madrid, Spain

\section{WILLIAM E. FRY}

Cornell University, Ithaca, USA

\section{JUNJIANG Fu}

Luzhou Medical College, Luzhou, China

\section{Alberto Gallifuoco}

University of L'Aquila, L'Aquila, Italy

IMAN A. Y. GHANNAM

National Research Centre, Cairo, Egypt 


\section{POORAN GOLKAR}

Isfahan University of Technology, Isfahan, Iran

\section{ANGel Gonzalez DElgado}

Universidad de Cartagena, Cartagena, Colombia

\section{JOANNA GRACZ}

Institut of Bioorganic Chemistry, PAS, Poznań, Poland

\section{DARIUSZ GRZEBELUS}

University of Agriculture in Krakow, Kraków, Poland

\section{EWA GRZEBELUS}

University of Agriculture in Krakow, Kraków, Poland

\section{Charu Gupta}

Amity University, Noida, India

DOROTA GURDA

Institute of Bioorganic Chemistry, PAS, Poznań, Poland

\section{TAO HE}

Qinghai University, Xining, China

\section{MOHSEN HESAMI}

University of Tehran, Teheran, Iran

\section{LAY HORNG-LIANG}

National Pingtung University of Science and Technology, Pingtung, Taiwan

\section{MOHAMMAD AMzAD Hossain}

University of Nizwa, Nizwa, Oman

XianglONG HU

South China Normal University Guangzhou, China

\section{MARZIYEH JAFARI}

Ramin University of Agriculture and Natural Resources, Khuzestan, Iran

\section{KAROLINA JARZYNIAK}

Poznan University of Life Sciences, Poznań, Poland

\section{PhILIPPE JEANDET}

University of Reims, Reims, France

\section{BYEONG-RYOOL JEONG}

Korea Advanced Institute of Science and Technology, Daejeon, Korea (South)

\section{NIRMAL JOSHEE}

Fort Valley State University, Fort Valley, USA

\section{ANDRZEJ KaLISZ}

University of Agriculture in Krakow, Kraków, Poland

\section{KAMNOON KANCHANAPOOM}

Prince of Songkla University, Songkhla, Thailand

\section{SUNDRAM KARUPIAH}

AIMST University, Semeling, Malaysia

\section{SUKHMINDERJIT KAUR}

Chandigarh University, India

\section{Purshotam KaushiK}

Gurukul Kangri University, India

\section{VICHIEN KITPREECHAVANICH}

Kasetsart University, Bangkok, Thailand

\section{ARUNA JYOTHI KORA}

National Centre for Compositional Characterisation of Materials, Hyderabad, India

\section{EDYTA KOSTRZEWA-SUSŁOW}

Wrocław University of Environmental and Life Sciences, Wrocław, Poland

ТомонІко KUво

Hokkaido University, Sapporo, Japan

\section{JAKUB KUCZYŃSKI}

Institute of Bioorganic Chem istry, PAS, Poznań, Poland

\section{VINOD KUMAR}

Uttaranchal University, Dehradun, India

\section{DHILIA UDIE LAMASUDIN}

Universiti Putra Malaysia, Serdang, Selangor, Malaysia

\section{MARZENA LEWANDOWSKA}

L. Rydygier Collegium Medicum in Bydgoszcz, Bydgoszcz, Poland

\section{KUAN-HUNG LIN}

Chinese Culture University, Taiwan

JACEK LIPOK

University of Opole, Opole, Poland

\section{ZHAN-YING LIU}

Inner Mongolia University of Technology, Hohhot, China

\section{LOPES FORTES RIBAS LUCIANA}

Federal University of Parana, Brazil, Brazil

TIANZHOU MA

University of Pittsburgh, Pittsburgh, USA

\section{ANUPAMA MAHAJAN}

Shaheed Udham Singh College of Engineering and Technology, Chandigarh, India 


\section{Ritu MAHAJAN}

University of Jammu, Jammu, India

\section{Elena M. Manaig}

Laguna State Polytechnic University, Laguna, Philippines

\section{LAXMan S. MEena}

CSIR - Institute of Genomics and Integrative Biology, Delhi, India

\section{MAHSA MOVAHEDI}

University of Sheffield, United Kingdom

\section{Chong Mun Keat}

Merck Singapore, Singapore

\section{DOROTA NAROŻNA}

Poznan University of Life Sciences, Poznań, Poland

\section{AMIR SASAN MOZAFFaRI NEJAD}

Hamadan University of Medical Sciences, Hamadan, Iran

\section{VINOD NIGAM NIGAM}

Birla Institute of Technology, Mesra, India

\section{NIK ABD RAHMAN}

Universiti Putra Malaysia, Selangor, Malaysia

\section{QIUHONG NIU}

Nanyang Normal University, Nanyang, China

\section{SAIFUDDIN NOMANBHAY}

The National Energy University, Kajang, Malaysia

\section{KATARZYNA NUC}

Poznan University of Life Sciences, Poznań, Poland

\section{MAGDOLEEN OSMAN}

National Centre for Research, Khartoum, Sudan

\section{Charles OsuJi}

Biotechnology Advance Research Center, Sheda, Nigeria

\section{HÜMEYRA ÖZKAN}

Kocaeli University, Kocaeli, Turkey

\section{RAJESh PAL}

Amity University, Uttar Pradesh, India

\section{Nita PAREKH}

International Institute of Information Technology, Gachibowli, India

\section{MARZENA POPIELARSKA-KONIECZNA}

Jagiellonian University, Kraków, Poland
A. S. V. Prasad

G.I.T.A.M Dental College, Rishikonda, India

\section{JOSHI RAMESH}

Samrat Prithviraj Chauhan Government College, Ajmer, India

\section{KATARZYNA ROLLE}

Institute of Bioorganic Chemistry PAS, Poznań, Poland

SUPRAVA SAHOO

Siksha ${ }^{\circ} O^{\circ}$ Anusandhan University, Bhubaneshwar, India

\section{TAWFiK A. SALEH}

King Fahd University of Petroleum and Minerals, Dhahran, Saudi Arabia

\section{SUREKHA K. SATPUTE}

Savitribai Phule Pune University, Pune, India

\section{ORATHAI SAWATDICHAIKUL}

Kasetsart University, Bangkok, Thailand

WOLF-DIETER SCHUBERT

University of Pretoria, Pretoria, South Africa

\section{MAREK SELWET}

Poznan University of Life Sciences, Poznań, Poland

\section{DEEPANSH SHARMA}

Lovely Professional University, Phagwara, India

\section{SARIKA SHARMA}

Arni University, Indora, Himachal Pradesh, India

\section{SUSHEEL SHARMA}

Sher-e-Kashmir University of Sciences and Technology of Jammu, Jammu, India

\section{UTKARSH SHEDBALKAR}

Institute of Science, Mumbai, India

\section{Agrawal Shweta}

Sanghvi Institute of Management and Science, Indore, India

\section{DEEPTI SINGH}

Guru Angad Dev Veterinary and Animal Sciences University, Ludhiana, India

SANDEEP SINGH

Maharshi Dayanand University, Rohtak, India

\section{ALeKSANDAR Kolev SLavov}

University of Food Technologies, Plovdiv, Bulgaria

ANNA T. STADLER

Johannes Kepler University Linz, Linz, Austria 


\section{MACIEJ STOBIECKI}

Institute of Bioorganic Chemistry PAS, Poznań, Poland

\section{XAVIER SURESH}

Sathyabama University, India

\section{HATÍRA TASSKÍN}

University of Niğde, Niğde, Turkey

\section{ROCKY THOKCHOM}

Uttar Banga Krishi Viswavidyalaya, Pundibari, India

\section{AgATA TyCZEWSKA}

Institute of Bioorganic Chemistry, PAS, Poznan, Poland

\section{Alicja Tymoszuk}

UTP University of Science and Technology, Bydgoszcz, Poland

\section{LAKSHMINARAYANA R. VEMIREDDY}

Acharya N. G. Ranga Agricultural University, Rajendranagar, India

\section{Prathibha Vyas}

Lovely Professional University, India

\section{YASIR WAHEED}

Foundation University Islamabad, Islamabad, Pakistan

\section{CONG WANG}

Nanjing Agricultural University, China

\section{KATARZYNA WOJCIECHOWICZ}

Adam Mickiewicz University, Poznań, Poland

\section{ELIZA WYSZKO}

Institute of Bioorganic Chemistry, PAS, Poznań, Poland

\section{RAJESH YARRA}

University of Hyderabad, Hyderabad, India

\section{SABINA YASMIN}

Agricultural University, Mymensingh, Bangladesh

\section{СHOTIKA YOKTHONGWATTANA}

Kasetsart University, Bangkok, Thailand

\section{ZHAOYANG YUAN}

Michigan State University, East Lansing, USA

\section{HUAWEI ZHANG}

Zhejiang University of Technology, Hangzhou, China 